\title{
ESTENOSES BENIGNAS DE ESÔFAGO: abordagem endoscópica com velas de Savary-Gilliard
}

\author{
Paula NOVAIS ${ }^{1}$, Eponina LEMME², Claudia EQUI ${ }^{1}$, Claudia MEDEIROS ${ }^{3}$, Camila LOPES $^{3}$ e \\ Cleber VARGAS²
}

\begin{abstract}
RESUMO - Racional - As estenoses benignas de esôfago são complicações decorrentes de diversas causas. Possuem tratamentos similares, na maioria dos casos necessitando de dilatação endoscópica, no entanto a resposta terapêutica, tempo ideal de tratamento, assim como intervalo entre as sessões podem ser variáveis. Objetivo - Analisar, do ponto de vista endoscópico, as estenoses benignas de esôfago em 14 anos de experiência no Hospital Universitário Clementino Fraga Filho da Universidade Federal do Rio de Janeiro, RJ, avaliando etiologia, a extensão da estenose, o número de dilatações necessário para atingir resposta terapêutica satisfatória, assim como a relação entre a extensão da estenose e a resposta terapêutica. Método - Foram analisadas 2.568 dilatações endoscópicas com uso de velas de Savary-Gilliard em 236 pacientes, durante um período de 14 anos e 10 meses, até junho de 2007. Resultados - A estenose péptica foi a causa mais freqüentemente encontrada, seguida pela estenose cáustica. As estenoses longas e cáusticas necessitaram de maior número de sessões para ausência de disfagia. Estenoses pépticas e curtas responderam melhor a número menor de sessões de dilatação. Conclusão - A estenose péptica foi a causa mais comum e respondeu bem à terapia endoscópica, em concordância com a literatura. As estenoses cáusticas foram as mais refratárias, principalmente as longas. Quanto maior foi a extensão da estenose, também maior foi o número de sessões necessárias. Estenoses curtas apresentaram boa evolução na maioria dos casos. O número de dilatações necessárias dependeu diretamente da causa e da extensão da estenose.
\end{abstract}

DESCRITORES - Estenose esofágica. Dilatação. Esofagoscopia.

\section{INTRODUÇÃO}

As estenoses benignas de esôfago são complicações decorrentes de diversas causas. Possuem tratamento semelhante, na maioria dos casos necessitando de dilatação endoscópica. No entanto a resposta terapêutica, tempo ideal de tratamento, assim como intervalo entre as sessões de dilatação podem ser variáveis, não havendo consenso na literatura a respeito deste fato.

Neste estudo retrospectivo, apresenta-se a experiência de 14 anos do Setor de Endoscopia Digestiva do Hospital Universitário Clementino Fraga Filho da Universidade Federal do Rio de Janeiro, RJ, em relação às estenoses benignas de esôfago.

\section{MÉTODO}

Foram analisadas 2.568 dilatações em 236 pacientes (109 mulheres e 127 homens com média de idade de 56 anos, variando de 3-88 anos), durante um período de 14 anos e 10 meses, até junho de 2007.
As dilatações foram realizadas em ambiente de ambulatório, com intervalos variáveis entre as sessões. O procedimento foi realizado com o paciente em jejum de no mínimo 8 horas, sob sedação consciente (diazepam e meperidina intravenosos) e oximetria contínua. Utilizouse um fio guia metálico flexível, introduzido através da estenose, sob visão endoscópica, sobre o qual foram passadas as velas de Savary-Gilliard, constituídas de material de polivinil maleável, com aumento gradual do calibre. O diâmetro das velas foi individualizado de acordo com o grau de estenose apresentado.

Foram avaliados dados como causa e extensão das estenoses. Estas foram classificadas como curtas se até $3 \mathrm{~cm}$, médias se entre 3 e $7 \mathrm{~cm}$ e longas quando com mais de $7 \mathrm{~cm}$ de extensão, sendo correlacionado o número de dilatações com sua extensão e etiologia. A influência da etiologia na extensão da estenose também foi analisada. O objetivo final do tratamento instituído era ausência ou redução acentuada da disfagia em vigência de calibre satisfatório de esôfago $(45 \mathrm{Fr}=15 \mathrm{~mm})$, não necessariamente alcançado através da vela de maior calibre $(60 \mathrm{Fr}=20 \mathrm{~mm})$.

Serviço de Gastroenterologia, Hospital Universitário Clementino Fraga Filho, Universidade Federal do Rio de Janeiro (HUCFF/UFRJ), RJ

1 Serviço de Gastroenterologia HUCFF/UFRJ; ${ }^{2}$ Departamento de Clínica Médica, Faculdade de Medicina da UFRJ; ${ }^{3}$ Faculdade de Medicina da UFRJ.

Correspondência: Dra. Paula Novais - Rua Jeronimo Mesuita - casa 154 - Barra da Tijuca - 22640-110 - Rio de Janeiro, RJ. E-mail: paulanovais@gmail.com 


\section{Análise estatística}

Para análise estatística dos dados foram utilizados os testes de coeficiente de contingência de Pearson (analisando a influência do sexo sobre a extensão da estenose) e ANOVA (análise de variância da influência da extensão e etiologia sobre o número de dilatações). O nível de significância empregado foi de $P<0,05$.

\section{RESULTADOS}

A estenose péptica foi a causa mais prevalente, ocorrendo em $43 \%$ dos pacientes, seguida da estenose cáustica apresentada por $16 \%$ dos pacientes (Tabela 1 ).

TABELA 1. Causa das estenoses benignas do esôfago (2.568 dilatações em 236 pacientes)

\begin{tabular}{lcc}
\hline Causa & $\mathrm{n}$ & $\%$ \\
\hline Péptica & 102 & 43,3 \\
Cáustica & 39 & 16,5 \\
Outras & 27 & 11,4 \\
Pós-escleroterapia & 24 & 10,2 \\
Pós-operatória & 22 & 9,3 \\
Indefinidas & 9 & 3,8 \\
Anel de Schatzki & 7 & 3,0 \\
Plummer-Vinson & 6 & 2,5 \\
Total & 236 & $100 \%$ \\
\hline
\end{tabular}

outras: infecciosas, actínica, pós uso de sonda nasogástrica, esofagite eosinofilica, pós-fundoplicatura, drogas

Em relação à extensão da estenose, foram avaliados 186 do total de 236 pacientes estudados e observou-se que cerca de $70 \%$ $(131 / 186)$ apresentaram estreitamentos curtos (Tabela 2), estes representados em sua maioria por estenoses pépticas (Tabela 3). Estenoses de extensão médias e longas foram mais freqüentes em pacientes cuja origem era cáustica (Tabelas 4 e 5).

TABELA 2. Extensão das estenoses (total analisado $=186$ pacientes)

\begin{tabular}{lcc}
\hline Extensão & $\mathbf{n}$ & $\%$ \\
\hline Curtas $(<3 \mathrm{~cm})$ & 131 & 70,4 \\
Médias $(3-7 \mathrm{~cm})$ & 38 & 20,4 \\
Longas $(>7 \mathrm{~cm})$ & 17 & 9,2 \\
\hline
\end{tabular}

TABELA 3. Causa das estenoses curtas (131 pacientes)

\begin{tabular}{lcc}
\hline Causa & $\mathbf{n}$ & $\%$ \\
\hline Péptica & 70 & 53,4 \\
Outras & 25 & 19,0 \\
Pós-escleroterapia & 18 & 13,7 \\
Pós-operatória & 13 & 10,0 \\
Cáustica & 5 & 3,9 \\
Total & 131 & $100 \%$ \\
\hline
\end{tabular}

TABELA 4. Causa das estenoses médias (38 pacientes)

\begin{tabular}{lcc}
\hline Causa & $\mathbf{n}$ & $\%$ \\
\hline Cáustica & 15 & 39,5 \\
Péptica & 13 & 34,2 \\
Outras & 5 & 13,2 \\
Pós-escleroterapia & 4 & 10,5 \\
Pós-operatória & 1 & 2,6 \\
Total & 38 & $100 \%$ \\
\hline
\end{tabular}

TABELA 5. Causa das estenose longas (17 pacientes)

\begin{tabular}{lcc}
\hline Causa & $\mathbf{n}$ & $\%$ \\
\hline Cáustica & 10 & 58,8 \\
Outras & 5 & 29,4 \\
Péptica & 2 & 11,8 \\
Pós-escleroterapia & 0 & 0 \\
Pós-operatória & 0 & 0 \\
Total & 17 & $100 \%$ \\
\hline
\end{tabular}

As estenoses pépticas foram predominantemente curtas, em 70 pacientes dos 85 analisados quanto à extensão (82\%) (Tabela 6). O oposto ocorreu nas estenoses cáusticas que foram predominantemente longas e médias (25 pacientes dos 30 analisados quanto à extensão $(83 \%))$. As estenoses decorrentes de escleroterapia de esôfago e cirurgias também foram curtas na sua maioria ( $82 \%$ e $93 \%$, respectivamente).

TABELA 6. Influência da causa na extensão da estenose

\begin{tabular}{lccc}
\hline Causa & $\begin{array}{c}\text { Estenose curta } \\
(\mathrm{n} / \%)\end{array}$ & $\begin{array}{c}\text { Estenose média } \\
(\mathrm{n} / \%)\end{array}$ & $\begin{array}{c}\text { Estenose longa } \\
(\mathrm{n} / \%)\end{array}$ \\
\hline Péptica $(\mathrm{n}=85)$ & $70 / 85(82 \%)$ & $13 / 85(16 \%)$ & $2 / 85(2 \%)$ \\
Cáustica $(\mathrm{n}=30)$ & $5 / 30(17 \%)$ & $15 / 30(50 \%)$ & $10 / 30(33 \%)$ \\
Pós-operatória $(\mathrm{n}=14)$ & $13 / 14(93 \%)$ & $1 / 14(7 \%)$ & $0 / 14$ \\
Pós-escleroterapia $(\mathrm{n}=22)$ & $18 / 22(82 \%)$ & $4 / 22(18 \%)$ & $0 / 22$ \\
Outras $(\mathrm{n}=35)$ & $25 / 35(72 \%)$ & $5 / 35(14 \%)$ & $5 / 35(14 \%)$ \\
Total $(\mathrm{n}=186)$ & $131 / 186(70,4 \%)$ & $38 / 186(20,4 \%)$ & $17 / 186(9,1 \%)$ \\
\hline
\end{tabular}

As estenoses pós-escleroterapia necessitaram de menos sessões de dilatações (cinco dilatações/paciente), seguida das pépticas, que necessitaram em média de 8,8 dilatações por paciente. As lesões cáusticas foram as que necessitaram de maior número de sessões, em média 24,6 dilatações por paciente (Tabela 7, Figura 1), apresentando diferença estatisticamente significante em relação às outras causas $(P<0,0000)$. Entretanto, analisando separadamente as causas pósoperatória, péptica e pós-escleroterapia, não se observou diferença significante entre o número médio de dilatações $(P=0,175667)$.

TABELA 7. Relação entre causa da estenose e o número de dilatações (236 pacientes)

\begin{tabular}{lc}
\hline Causa & $\mathrm{N}^{\circ}$ de dilatações por paciente \\
\hline Cáustica & $24,6 *$ \\
Pós-operatória & $8,9^{* *}$ \\
Péptica & $8,8^{* *}$ \\
Pós-escleroterapia & $5,1 * *$ \\
\hline${ }^{* A N O V A-P<0,000001}$ & \\
$* \star A N O V A-P=0,175667$ &
\end{tabular}

Estreitamentos de segmento longo necessitaram em média 28,9 dilatações por paciente, enquanto que estreitamentos de curta extensão necessitaram de cerca de 7,6 dilatações por paciente $(P<0,00001)$ (Tabela 8, Figura 2).

Nas 2.568 dilatações realizadas, ocorreram três perfurações, $\mathrm{o}$ que representa $0,1 \%$ do total. Duas destas foram microperfurações em terço médio, em esofagites cáusticas, tratadas clinicamente, com alta hospitalar em 48 horas. A terceira perfuração ocorreu em esôfago cervical, em estenose de causa indefinida, tratada com esofagostomia, evoluindo com boa recuperação. Não houve sangramento maior que o habitual ou mortalidade nesta casuística. 
TABELA 8. Relação entre a extensão da estenose e o número de dilatações

\begin{tabular}{ccc}
\hline Extensão & $\mathrm{N}^{\circ}$ de dilatações por $\mathrm{n}^{\circ}$ de pacientes & $\mathrm{N}^{\circ}$ de dilatações por paciente \\
\hline Até $3 \mathrm{~cm}$ & 993 dilatações/131 pacientes & 7,58 dilatações/pct* \\
$3-7 \mathrm{~cm}$ & 715 dilatações $/ 38$ pacientes & 18,8 dilatações/pct* \\
$>7 \mathrm{~cm}$ & 484 dilatações $/ 17$ pacientes & 28,4 dilatações/pct* \\
\hline
\end{tabular}

${ }^{*}$ ANOVA $=$ significância entre as diferenças das médias $(P=0,000001)$

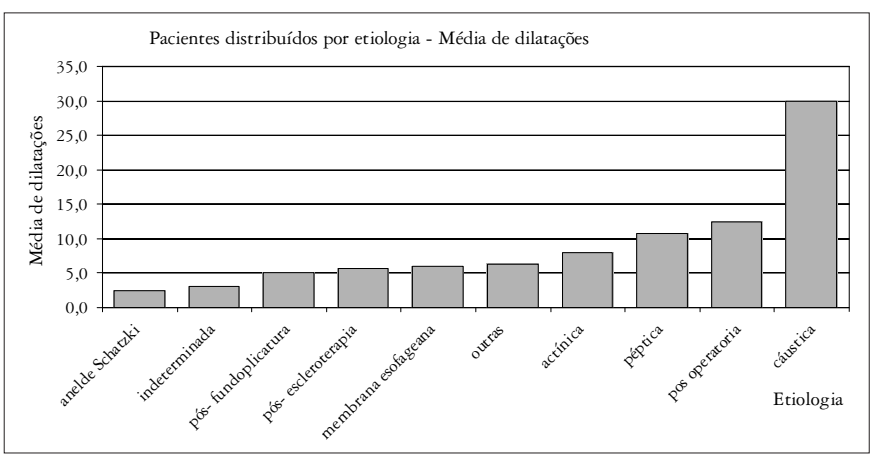

FIGURA 1. Média de dilatações por etiologia

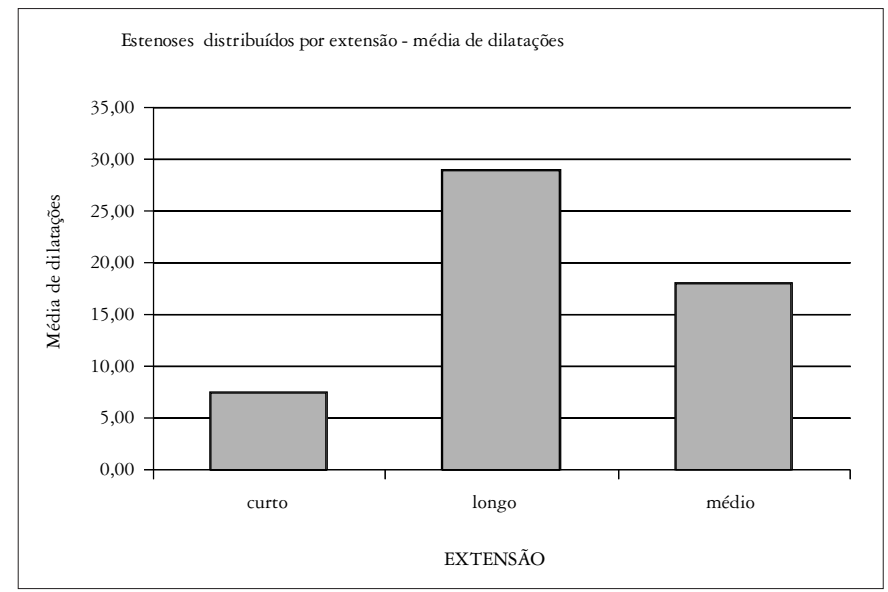

FIGURA 2. Média de dilatações por extensão

\section{DISCUSSÃO}

As estenoses benignas do esôfago podem ser causadas por diferentes situações. Na literatura, assim como no presente estudo, a causa mais comum é a estenose péptica ${ }^{(17)}$ causada pelo refluxo gastroesofágico crônico, que corresponde a 60\%-70\% dos $\operatorname{casos}^{(2,6,20,23)}$. Outras causas são a ingestão acidental ou proposital de substâncias corrosivas, o uso prolongado de sonda nasogástrica ou nasoenteral ${ }^{(12)}$, os anéis e membranas esofagianas, radioterapia, pós-escleroterapia de varizes ${ }^{(9)}$, esofagite infecciosa, esofagite eosinofílica, traumas de esôfago que podem levar ao desenvolvimento de hematomas intra-murais ou interrupção da nutrição ${ }^{(19)}$, sarcoidose, injúria elétrica externa ${ }^{(7)}$ e outros.

O diagnóstico causal da estenose pode ser definido em $80 \%$ dos casos apenas através da anamnese. A esofagografia contribui muito para definir o grau de estenose, localização, presença de tortuosidade e escolha do tipo de dilatador a ser empregado, sendo geralmente considerado como o exame inicial. A endoscopia digestiva alta é imprescindível, pois avalia a mucosa da região afetada, exclui estenoses malignas e possibilita a realização de biopsias, que devem ser realizadas mesmo quando o diagnóstico etiológico for evidente ${ }^{(4)}$. A ecoendoscopia raramente é necessária nas estenoses benignas, mas a esofagomanometria pode ser útil nos casos em que se quer definir se houve também acometimento motor do órgão, como no caso das estenoses cáusticas profundas ${ }^{(8,22)}$.

Estima-se que 7\% a 23\% dos pacientes com doença do refluxo gastroesofágico (DRGE) evoluam com estenose esofagiana ${ }^{(3)}$. Como apresentado no presente estudo, são estenoses geralmente anelares $(<1 \mathrm{~cm})$ e tipicamente localizadas no segmento distal. Respondem muito bem à terapia de dilatação com sucesso maior que $80 \%{ }^{(5)}$, porém cerca de $30 \%$ dos pacientes apresentam recurrência da disfagia, necessitando de outras dilatações. A terapia com inibidores de bomba de prótons (IBP) em dose plena e medidas anti-refluxo devem sempre ser realizadas. É preciso avaliar indicação de correção cirúrgica da DRGE com fundoplicatura gástrica ${ }^{(23)}$.

Agentes corrosivos, quando ingeridos acidental (crianças em sua maioria) ou propositalmente (adultos em tentativas de suicídio) podem causar estenoses esofagianas, que constituem as de maior risco de perfuração e que mais apresentam recurrência ${ }^{(5,20)}$. O exame endoscópico na fase aguda pode indicar o prognóstico. TIRYAKI et al. ${ }^{(26)}$ observaram melhor resposta terapêutica quando as dilatações eram iniciadas na fase aguda, 7 dias após ingestão do corrosivo. A extensão do segmento afetado também é fator prognóstico. Na presente casuística, observa-se que, quanto mais extensa foi a estenose, necessitou-se maior número de dilatações. Após o desaparecimento dos sintomas, o acompanhamento deve ser realizado de 3 em 3 meses no primeiro ano e depois anualmente ${ }^{(7)}$. TEMIR et al. ${ }^{(25)}$ defendem a realização de novas dilatações apenas quando houver reaparecimento da disfagia

Pacientes submetidos a cirurgias de esôfago, principalmente as esofagectomias e até mesmo a cirurgias de correção de hérnia de hiato, podem desenvolver estenoses, seja por desenvolvimento de fístulas ou isquemia local, uso de "stapler" ou pelo próprio processo evolutivo cicatricial. O índice de sucesso das dilatações endoscópicas nestes pacientes fica em torno de $75 \%$ a $92 \%$, geralmente necessitando de cerca de três a cinco sessões para resolução dos sintomas ${ }^{(13)}$. No levantamento desta casuística verificou-se que pacientes com estenoses pós-operatórias necessitaram em média de nove sessões para boa resposta. Recomenda-se iniciar a dilatação após 2 semanas de cirurgia, sendo 2 meses, o ideal ${ }^{(13)}$

Sabe-se que em apenas 24 horas do uso de sonda nasogástrica, pode-se observar edema intra-epitelial e hemorragia submucosa no esôfago e se a sonda permanecer por mais de 5 dias, nota-se além de hiperemia, presença de ulcerações. Isto ocorre tanto pela irritação local direta da sonda, como também pela facilitação do refluxo gastroesofágico. Um paciente da presente série apresentou importante estenose longa decorrente do seu uso, necessitando de 36 sessões de dilatação para que fosse alcançado e mantido o calibre de $60 \mathrm{Fr}$. 
A radioterapia pode causar estenose por dano direto ao DNA e produção de radicais livres que estimulam morte celular. A incidência gira em torno de $25 \%$ a $67 \%$ nos paciente irradiados para tumor primário e de $1 \%$ a $20 \%$ para irradiação de tumores adjacentes (mama, pulmão, tireóide) ${ }^{(13)}$. Geralmente é necessária irradiação de mais de 6.000 rads fracionados. No presente trabalho, a estenose actínica foi incomum, ocorrendo em apenas quatro pacientes $(1,69 \%)$ que necessitaram, em média, de 14,5 sessões de dilatação para alcançar resposta terapêutica.

A esofagite eosinofílica, entidade predominante em crianças, na qual existe inflamação crônica do esôfago, caracterizada por infiltração de mais de 20-24 eosinófilos por campo de grande aumento, detectada pelo estudo histopatológico, é causa ainda subdiagnosticada em adultos com estenose de esôfago. $\mathrm{O}$ tratamento inclui dilatações endoscópicas e afastamento do fator desencadeante (geralmente aeroalérgenos e alérgenos alimentares) e corticóide tópico. No presente estudo avaliaram-se dois pacientes com este diagnóstico, que necessitaram, em média, de sete sessões de dilatação para remissão da disfagia.

Esofagite causada por ingestão de medicamentos em forma de pílulas é causa infreqüente de estenose. Medicações como quinidina, tetraciclina, antiinflamatórios não-hormonais, cloreto de potássio e alendronato podem causar estenose por lesão direta à mucosa devido ao contato prolongado, diminuição da atividade motora, diminuição da depuração esofagiana, síndrome de Stevens-Johnson e alteração da coagulação. Neste estudo, não foi possível confirmar o uso de drogas como fator causador de estenose, embora tenha se levantado suspeita em quatro casos.

Membranas e anéis são causas de estenoses que respondem muito bem às dilatações endoscópicas. No caso dos anéis, de Schatzki, $68 \%$ dos pacientes permanecem sem disfagia por período de 1 ano após a primeira dilatação, porém muitos precisam de novas dilatações no decorrer da vida (apenas $11 \%$ não recorrem em 5 anos $)^{(5)}$. No presente estudo avaliaram-se sete pacientes com anel de Schatzki e seis com membrana, todos evoluindo com boa resposta à terapêutica endoscópica (2,1 e 6 dilatações por paciente, respectivamente).

Correlacionando-se a extensão da estenose com sua causa, verificou-se que as causas têm um comportamento próprio. Assim, as estenoses pépticas são geralmente curtas e as cáusticas comprometem, na maior parte da vezes, segmentos longos e médios.

Quando comparado o número de dilatações realizadas com a causa e a extensão da estenose, verificou-se que lesões curtas ou anelares, como as pós-operatórias e pépticas, podem ser tratadas através de menor número de dilatações. Já as estenoses cáusticas, geralmente longas, necessitaram de número bastante elevado de sessões. PEREIRA-LIMA et al. ${ }^{(18)}$ estudaram 1.043 dilatações realizadas em 153 pacientes com estenoses benignas de esôfago de diversas causas (predominantemente pós-operatórias, que corresponderam a $57 \%$ do total) e acharam resultados semelhantes em relação às estenoses pépticas (média de três sessões de dilatação para alcançar calibre esofagiano de 42Fr). Entretanto, quanto às estenoses cáusticas, foi necessário número de dilatações menor que o referido pelos autores desta série $(5,0 \times 24,9$ dilatações por paciente).
A decisão do tipo de dilatador a ser utilizado depende da disponibilidade do material e experiência de cada serviço. No Serviço onde este estudo foi realizado, são habitualmente utilizadas as velas de Savary-Gilliard, constituídas de material polivinil maleável e progredidas através da estenose orientada pela passagem de um fio guia metálico. O uso da fluoroscopia durante a dilatação, assim como no hospital onde avaliou-se esta casuística, tem sido dispensado na maioria dos Serviços, mas pode ser útil nos casos de estenoses tortuosas, pseudodivertículos e hérnias de hiato volumosas, oferecendo maior segurança ao procedimento por permitir total controle do posicionamento do dilatador e do fio guia.

Não existe consenso em relação ao seguimento destes pacientes. MOURA et al. ${ }^{(14)}$ sugerem que as dilatações devem ser semanais no $1^{\circ}$ mês, quinzenal no $2^{\circ}$, de 21 em 21 dias nos $3^{\circ}$ e $4^{\circ}$ meses e mensal nos $5^{\circ}, 6^{\circ}$ e $7^{\circ}$ meses. O paciente seria, então, orientado a retornar se ainda relatasse os sintomas ou se houvesse retorno da disfagia, sendo considerado o caso como refratário, se estas queixas ocorressem em período inferior a 3 meses após a última dilatação. Alguns autores ${ }^{(18)}$ recomendam que o paciente deva ser mantido em programa de dilatação com intervalos curtos (semanal ou quinzenal) no início da terapia, até que seja alcançado como objetivo final, a ausência da disfagia; outros $^{(21)}$, porém, defendem a progressão até vela maior que $45 \mathrm{Fr}$, mesmo que o paciente já esteja assintomático ${ }^{(27)}$. No Serviço dos autores, este acompanhamento foi variável. Na maior parte dos casos, os pacientes eram mantidos em programa de dilatação semanal inicial e depois quinzenal, até a ausência de disfagia em vigência de um calibre satisfatório de esôfago (45Fr). Muitos pacientes não necessitaram alcançar a maior vela (60Fr) para obter ausência de sintomas.

Entre as complicações da dilatação, a perfuração ocorre em $0,5 \%$ a $1,2 \%{ }^{(2,21)}$ e sangramento maior que habitual, podem ocorrer em menos de $0,5 \%$ dos $\operatorname{casos}^{(16)}$, bacteremia em $20 \%$ $45 \%$, porém infecções são raras, havendo relato de abscesso cerebral pós-dilatação ${ }^{(3)}$. No presente estudo, houve três casos de perfuração $(0,1 \%$ do total de dilatações $)$, tratados com conduta conservadora em dois e esofagostomia em um, comprovando que o procedimento é muito seguro.

O uso de corticóide (triamcinolona) no local da estenose ou intralesional (nas lacerações após dilatação) é utilizado em alguns centros médicos, pois acredita-se que ao inibir a síntese de colágeno, possa se diminuir o número de sessões e aumentar o intervalo entre elas ${ }^{(10,11,15,24)}$. O intervalo preconizado para as injeções é de uma vez por semana durante o período de 1 mês ${ }^{(1)}$. Alguns pacientes deste estudo foram submetidos a injeções de corticóide intralesional e aparentemente tiveram benefícios, aumentando o intervalo necessário entre as sessões, porém estes resultados não puderam ser analisados, uma vez que não foi utilizado protocolo para seu uso.

\section{CONCLUSÃO}

No presente estudo, a estenose péptica foi a causa mais comum de estenoses benignas do esôfago e respondeu bem à terapia endoscópica em concordância com a literatura. As estenoses 
cáusticas foram as mais refratárias, principalmente as de longa extensão. Quanto maior foi a extensão da estenose, maior foi o número de sessões necessárias. Estenoses curtas apresentaram boa evolução na maioria dos casos. O número de dilatações necessárias dependeu diretamente da causa e da extensão da estenose.

\section{AGRADECIMENTO}

Ao Prof. Maurício de Pinho Gama da Divisão de Pesquisa do Hospital Universitário Clementino Fraga Filho, UFRJ, pelos cuidados com a análise estatística.

Novais P, Lemme E, Equi C, Medeiros C, Lopes C, Vargas C. Benign strictures of the esophagus: endoscopic approach with Savary-Gilliard bougies. Arq Gastroenterol. 2008;45(4):290-4.

ABSTRACT - Background - Benign esophageal strictures are complications that result from different causes. They are usually similarly approached, most of the cases needing endoscopic dilation. However the response to therapy, optimal timing for treatment and interval between sessions can vary. Aim - The authors evaluate 14 years of experience with benign stricture of the esophagus from the endoscopic point of view in the "Clementino Fraga Filho" University Hospital, Federal University of Rio de Janeiro, RJ, Brazil. They evaluated etiology, length of stricture, number of dilations needed to reach satisfactory therapeutic response, and the relation between length of stricture and therapeutic response. Methods - We analyzed 2,568 endoscopic dilations using Savary-Gilliard dilators in 236 patients. The follow up period was 14 years and 10 months, until June of 2007. Results - Peptic strictures were the more frequent, followed by caustic strictures. Long strictures and caustic strictures needed more sessions to abolish dysphagia. Peptic strictures and short ones had better response to a smaller number of sessions. Conclusion - In this study, peptic strictures were the commonest etiology and responded best to endoscopic therapy, in accordance with published literature. Caustic strictures were the most refractory, mainly the long segments. The longer the extension of stenosis, the greater was the number dilation sessions needed for relief. Short strictures had a good prognosis in the great majority of cases. The number of dilations depended directly on the etiology and the extension of the stricture.

HEADINGS - Esophageal stenosis. Dilatation. Esophagoscopy.

\section{REFERÊNCIAS}

1. Altintas E, Kacar S, Tunc B, Sezgin O, Parlak E, Altiparmark E, Saritas U, Salrin B. Intralesional steroid injection in benign esophageal strictures resistant to bougie dilation. J Gastroenterol Hepatol. 2004;19:1388-91

2. Andreollo NA, Lopes LR, Inogutti R, Brandalise NA, Leonardi LS. Conservative treatment of benign esophageal strictures using dilation. Analysis of 500 cases. Rev Assoc Med Bras. 2001;47:236-43.

3. Angel C, Wrenn E, Lobe T. Brain abcess: an unusual complication of multiple esophageal dilatations. Pediatr Surg Int. 1991;6:42-3.

4. Barkin JS, Taub S, Rogers AI. The safety of combined endoscopy, biopsy, and dilation in esophageal strictures. Am J Gastroenterol. 1981;76:23-6.

5. Deviere J. Dilation procedures. In: Tytgat GNJ, Classen M, Waye JD, Nakazawa S, editors. Practice of therapeutic endoscopy. 2nd ed. London: WB Saunders; 2000. p.29-37.

6. Drabek J, Keil R, Namesny I. The endoscopic treatment of benign esophageal strictures by balloon dilatation. Dis Esophagus. 1999;12:28-9.

7. Hassan SI, Uma Devi M, Kumar PS, Kumar BR, Reddy KV, Nageshwar K. Esophageal stricture following electrical injury. Indian J Gastroenterol. 2007;26:40-1.

8. Kahrilas PJ, Clouse RE, Hogan WJ. American Gastroenterological Association technical review on the clinical use of esophageal manometry. Gastroenterology. 1995; 109:1865-84.

9. Kochhar R, Goenka MK, Mehta SK. Esophageal strictures following endoscopic variceal sclerotherapy. Antecedents, clinical profile and management. Dig Dis Sci. 1992;37:347-52.

10. Kochhar R, Ray JD, Sriram PV, Kumar S, Singh K. Intralesional streoids augment the effects of endoscopic dilatation in corrosive esophageal strictures. Gastrointest Endosc. 1999;49:509-13.

11. Kochhar R, Makharia GK. Usefulness of intralesional triamcinolone in treatment of benign esophageal strictures. Gastrointestinal endosc. 2002;56:829-34.

12. Matugma SE, Sakai P, Ishioka S. Passagem de sonda para alimentação. In: Sakai P, Ishioka S, Filho FM, editores. Tratado de endoscopia digestiva diagnóstica e terapêutica. São Paulo: Atheneu; 1999. p.39-47.

13. Miyajima NT, Ueda SS, Sakai P. Estenose das anastomoses cirúrgicas do esôfago. In Sakai P, Ishioka S, Filho FM, editores. Tratado de endoscopia digestiva diagnóstica e terapêutica. São Paulo: Atheneu; 1999. p.95-101.

14. Moura EG, Maluf F, Chaves D. Esofagite de refluxo e estenose péptica. In Saka P, Ishioka S, Filho FM, editores. Tratado de endoscopia digestiva diagnóstica e terapêutica. São Paulo: Atheneu; 1999. p.65-71.
15. Moura EG, Ferrari S, Maluf F. Injeção de antiinflamatório esteróide. In: Sakai P, Ishioka S, Filho FM, editores. Tratado de endoscopia digestiva diagnóstica e terapêutica. São Paulo: Atheneu; 1999. p.33-8

16. Nostrant TT, Nandi OS. Esophageal dilatation. Gastroenterologist. 1998;6:5-15.

17. Patterson DJ, Graham DY, Smith JL, Schawartz JF, Alpert E, Lanza FL, Cain GD. Natural history of benign esophageal stricture treated by dilatation. Gastroenterology. 1983;85:346-50.

18. Pereira-lima JC, Ramires RP, Zamin I Jr, Cassal AP, Marroni CA, Mattos AA. Endoscopic dilatation of benign esophageal strictures: report on 1043 procedures. Am J Gastroenterol. 1999;94:1497-501.

19. Pineau BC, Ott DJ. Isolated proximal esophageal injury from blunt trauma: endoscopic stricture dilatation. Dysphagia. 2003;18:263-6.

20. Piotet E, Escher A, Monnier P. Esophageal and pharyngeal strictures: report on 1,862 endoscopic dilatations using the Savary-Gilliard technique. Eur Arch Otorhinolaryngol. 2008;265:357-64

21. Saeed ZA, Winchester CB, Ferro PS, Michaletz PA, Schwartz JT, Graham DY Prospective randomized comparison of polyvinyl bougies and through-the-scope balloons for dilation of peptic strictures of the esophagus. Gastrointest Endosc. 1995;41:189-95.

22. Singhal S, Kar P. Management of acid - and alkali-induced esophageal strictures in 79 adults by endoscopic dilation: 8-years' experience in New Delhi. Dysphagia. 2007;22:130-4.

23. Spechler SJ. AGA technical review in treatment of patients with dysphagia caused by benign disorders of the distal esophagus. Gastroenterology. 1999;117:233-54.

24. Streeter BL. Intralesional steroid injection therapy. Gastroenterol Nurs. 1999;22:7-9.

25. Temir GZ, Karkiner A, Karaça I, Ortaç R, Ozdamar A. The effectiveness of sucralfate against stricture formation in experimental corrosive esophageal burns. Surg Today. 2005;35:617-22.

26. Tiryaki T, Livanelioglu Z, Atayurt H. Early ougienage for relief of stricture formation following caustic esophageal burns. Pediatr Surg Int. 2005;21:78-80.

27. Zahid AS, Winchester C, Ferro P, Michaletz P, Schwartz J, Graham D. Prospective randomized comparison of polyvinyl bougies and through-the-scope balloons for dilation of peptic strictures of the esophagus. Gastrointest Endosc. 1995;41:189-95. 